JPE 10-2-15

\title{
Design Considerations of a Lithium Ion Battery Management System (BMS) for the STSAT-3 Satellite
}

\author{
Kyung-Hwa Park ${ }^{\dagger}$, Chol-Ho Kim*, Hee-Keun Cho**, and Joung-Ki Seo** \\ $\dagger^{* *}$ Satellite Technology Research Center, KAIST, Daejeon, Korea \\ ${ }^{*}$ Dept. of Electrical Engineering and Computer Science, KAIST, Daejeon, Korea
}

\begin{abstract}
This paper introduces a lithium ion battery management system (BMS) for the STSAT-3 satellite. The specifications of a lithium ion battery unit are proposed to supply power to the satellite and the overall electrical and mechanical designs for a lithium ion battery management system are presented. The structural simulation results will be shown to confirm the behavior of both the BMS and the cells.
\end{abstract}

Key Words: Battery Management System, Lithium Ion Battery, Power Density, State of Charge (SOC), State of Health (SOH)

\section{INTRODUCTION}

Traditionally, Ni-Cd and Ni-H2 batteries were used as space batteries [1], [2]. These days, lithium ion batteries are adapted for use as space batteries because of their high specific energy and energy density. They offer a low self discharge rate, long cycle life and a wide operation temperature range as shown in table 1 [3], [4]. These merits make them attractive for weight and volume sensitive application like satellite systems.

TABLE I

COMPARISON OF DIFFERENT BATTERY

\begin{tabular}{ccccc}
\hline Category & $\begin{array}{c}\text { Energy density } \\
(\mathrm{Wh} / \mathrm{L})\end{array}$ & $\begin{array}{c}\text { Specific energy } \\
(\mathrm{Wh} / \mathrm{kg})\end{array}$ & $\begin{array}{c}\text { Nominal } \\
\text { Voltage }(\mathrm{V})\end{array}$ & $\begin{array}{c}\text { Cycle life } \\
(\text { Time })\end{array}$ \\
\hline Ni-Cd & 100 & 35 & 1.2 & $500 \sim 2000$ \\
Lead acid & 70 & 35 & 2.0 & $200 \sim 700$ \\
Ni- MH & 240 & 75 & 1.2 & $300 \sim 600$ \\
Lithium & 400 & 150 & 3.7 & $>2000$ \\
\hline
\end{tabular}

The Science and Technology Satellite (STSAT) project is for developing small satellites with payloads for scientific missions. STSAT- 1 and STSAT-2 used Ni-Cd batteries but the newer STSAT-3 uses a lithium ion battery as an energy storage source. Fig. 1 shows the overall configuration and interface of the STSAT-3 electrical power subsystem (EPS).

Battery charging on the STSAT-3 is carried out by a solar power regulator (SPR) using 2 different modes: the peak

\footnotetext{
Manuscript received Aug. 10, 2009; revised Jan. 27, 2010

$\dagger$ Corresponding Author: ragm@satrec.kaist.ac.kr

Tel: +82-42-350-8607, Fax: +82-42-861-0064, KAIST

* Dept. of Electrical Engineering and Computer Science, KAIST, Korea

** Satellite Technology Research Center, KAIST, Korea
}

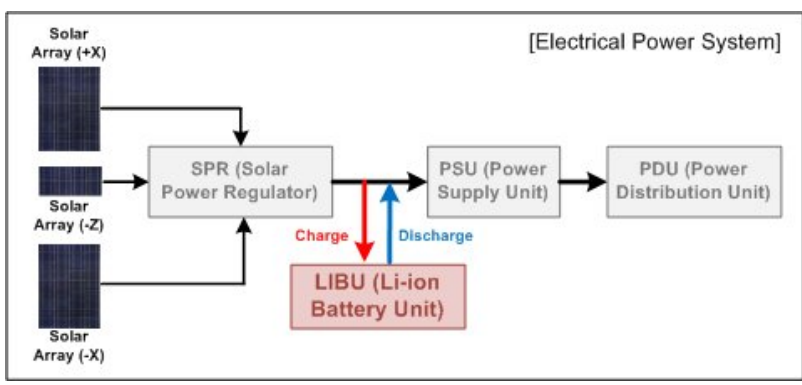

Fig. 1. Block diagram of STSAT-3 EPS.

power tracking mode and the voltage limit mode. Peak power tracking is utilized at the beginning of each satellite orbit until the selected charge voltage level is reached. At this point, the SPR enters the voltage limit mode, also known as the taper charge mode.

The SPR supplies power directly to the bus system and pay loads while in the sunlight. The lithium ion battery supplies power during eclipses or a lack of solar power. A power supply unit (PSU) generates various voltages for the satellite, a power distribution unit (PDU) turns on switches by commands from the tele command and telemetry module (TCTM) and the units and payloads finally operate.

As the chemical substances of the batteries change, the system structure changes. Lithium ion batteries are highly volatile and can cause serious damage and injury. A high temperature flame can be ejected for several meters if the temperatures or voltages of the cells go beyond their specified values [5]. Therefore, the battery management system for a lithium ion battery is essential to get rid of these battery failure 
hazards [6]-[12].

When employing a Li-ion battery cell in space, electrical and mechanical designs are important. A well thought out electrical design should be implemented for the cell balancing module. Since electrical design is closely related to the SPR and PSU, the power flow has to be controlled, the battery cell voltage has to be monitored and balanced and the equalization algorithm and circuit design are very considerable. Also, the mechanical design is essential to maintain the stability of the structure under the harsh thermal, vacuum, and vibration conditions found in space.

In this paper, the electrical and mechanical design of a BMS for the STSAT-3 lithium ion battery unit is researched. The BMS system is designed, considering the power capability, the SPR and PSU as well as the mechanical specification. Also, the battery control unit is designed for controlling the balancing circuit and communicating with the satellite system. Furthermore, since the BMS system is to be used in space, mechanical designs and tests are implemented. To prove the validity of the electrical and mechanical designs for the BMS, the experimental results are shown with the real STSAT-3 system.

\section{IElectrical Design Consideration Of The BMS}

\section{A. The specifications of the lithium ion battery}

The capacity of the battery is derived from the following equation:

$$
A h=\frac{P_{\text {eclips }} \times h r s_{\text {Disc }}}{V_{\text {nominal }} \times D O D \times F_{\text {Deg. }}}+\text { Margin }
$$

where,

$P_{\text {eclips }}:$ BOL power for the eclipse (Watt).

$h r s_{\text {Disc }}$ : Eclipse period (Hour).

$V_{\text {nominal }}$ : Nominal bus voltage $(\mathrm{V})$.

$F_{\text {Deg. }}$ : Degradation factor of the cells $(\% / 100)$.

$D O D$ : Depth of discharge $(\% / 100)$.

The STSAT-3 has an orbit with a period of 99.88 minutes and 35 minutes of maximum eclipse duration. The NCP 25-1 lithium ion cells, $30 \mathrm{Ah}$, which make up the battery pack were selected because it needs $209.65 \mathrm{~W}$ during the eclipse period. 8 cells were joined in series for generating $28.8 \mathrm{~V}$ of nominal bus voltage. Table 2 shows specifications of the STSAT-3 lithium ion battery pack.

TABLE II

ELECTRICAL SPECIFICATIONS OF STSAT-3 LI-ION BATTERY PACK

\begin{tabular}{cc}
\hline Item & Value \\
\hline Operating voltage (Battery) & $24 \sim 32.8 \mathrm{~V}$ \\
Operating voltage (Cell) & $3.0 \sim 4.1 \mathrm{~V}$ \\
Capacity (Battery) & $888 \mathrm{WHr}$ \\
Capacity (Cell) & $30 \mathrm{Ah}$ \\
Structure & 8 Series / 1Parallel \\
\hline
\end{tabular}

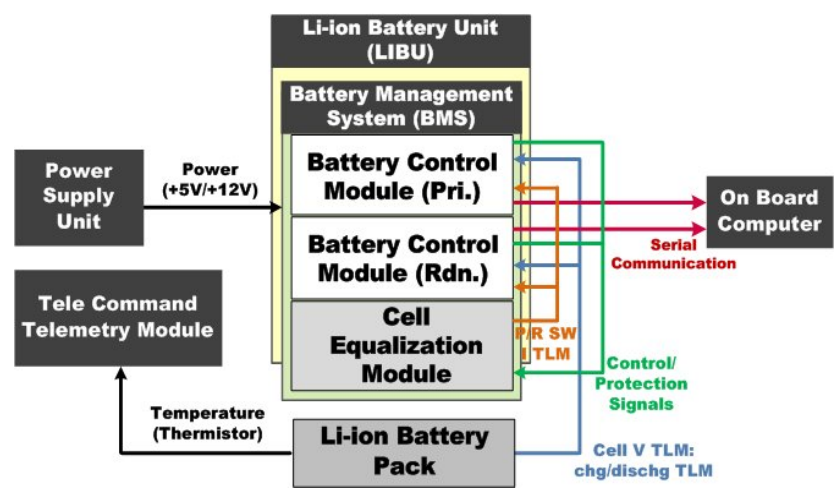

Fig. 2. Configuration of lithium ion battery unit for STSAT-3.

\section{B. The BMS configuration}

The BMS of the STSAT-3 includes three modules, the primary battery control module (BCM), the redundant $\mathrm{BCM}$ and the cell equalization module (CEM), as shown in Fig. 2.

The BCMs are in charge of controlling the CEM, processing telemetries and transmitting data through the RS422 serial buses to the on board computers (OBC). The CEM is turned on when the gap between the minimum and maximum voltages among the 8 battery cells is higher than the SOC $\pm 5 \%$. The CEM balances the cell voltages using the active balancing method and the topology is an improved fly back converter.

1) Battery control module (BCM): The BMS for the STSAT-3 should perform the following functions:

- Measuring the voltage and current of the battery pack during the charging and discharging periods.

- Measuring the temperature of each cell.

- Determining the state of each cell for likely failure.

- Controlling and protecting the cell equalization module (CEM).

- Performing and maintaining all communications with the on board computer ( $\mathrm{OBC}$ ) and the tele command telemetry module (TCTM).

The PSU supplies $+5 \mathrm{~V}$ and $+12 \mathrm{~V}$ of power to the primary and redundant BCM. For driving the micro-processor, each $\mathrm{BCM}$ has regulators to generate $+3.3 \mathrm{~V}$ of $\mathrm{I} / \mathrm{O}$ voltage and $+1.5 \mathrm{~V}$ of core voltage. At the incipient stage of operation, the primary $\mathrm{BCM}$ works and the redundant one stop operating because the regulators in the redundant $\mathrm{BCM}$ are disabled. Then if the hardware circuit detects an abnormal core voltage range, the primary BCM is turned off and the redundant $\mathrm{BCM}$ is turned on at the same time. Fig. 3 shows the autonomous change from the primary to the redundant BCM.

The micro-processor acquires cell voltage telemetries as shown in Fig. 4. Differential amplifiers are used to get each cell voltage. The 8 outputs of the op-amps become a single channel by a multiplexer. The signal enters the micro-processor after digitalization by an analog to digital converter. To enhance the accuracy of the differential amplifier, high-precision resistors with tolerances of $0.1 \%$ were used in manufacturing the qualification model. The current telemetries were obtained in the same manner.

The BCMs obtain each cell's voltage, the charge/discharge current of the battery pack, and the switch current of the cell 


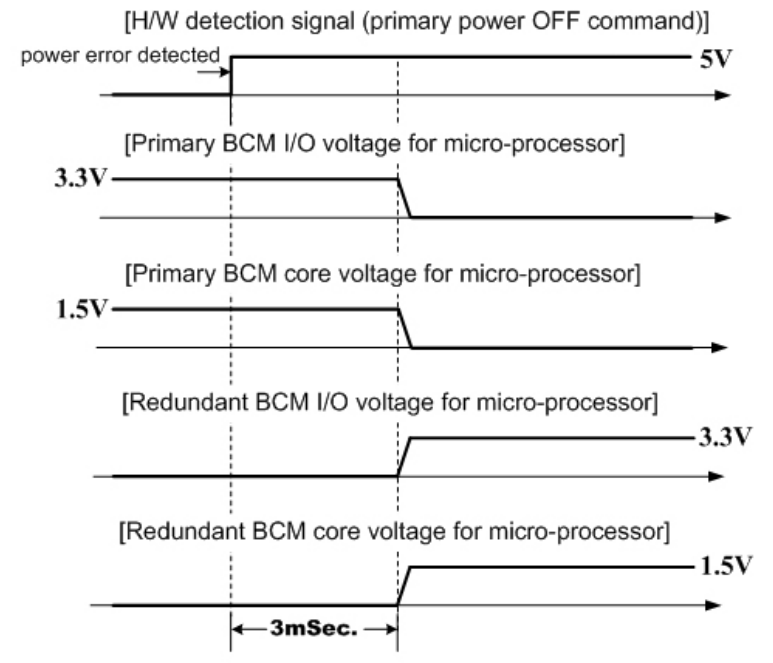

Fig. 3. Autonomous change from the primary BCM to the redundant BCM by a hardware detection signal.

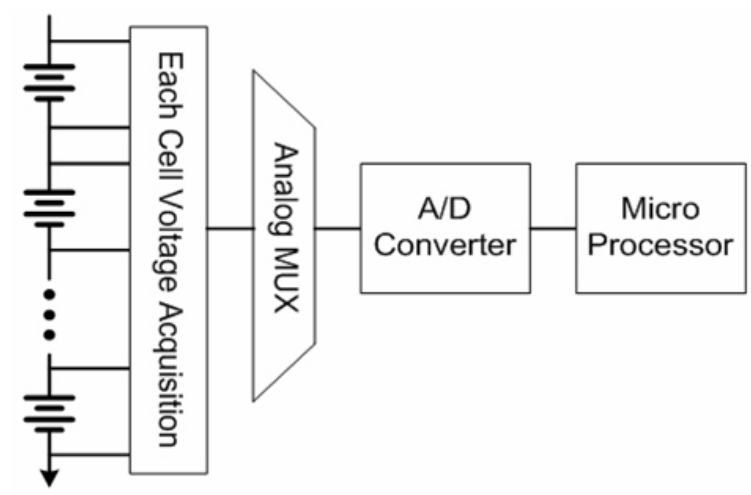

Fig. 4. The telemetry acquisition of BCM.

equalization module (CEM) for over current protection. To transmit them, the BCMs use two kinds of serial communication protocols. First, RS422 serial communication buses are used to communicate with the OBCs. Each of the BCMs sends data packets to the primary and redundant $\mathrm{OBCs}$ respectively. The BCMs use almost no internal memory because the OBC $\operatorname{logs}$ data every $16 \mathrm{sec}$ via the RS422 buses. The OBC holds a day's worth of data and then forwards them to a ground station (GS) when the satellite points to the earth. Second, an RS232 serial communication port is used for local debugging. The total raw data bits, $D_{\text {raw }}$, saved in the OBC are expressed as:

$$
D_{\text {raw }}=B_{\text {ch }} \times N_{\text {ch }} \times N_{\text {Orbit }} \times N_{\text {trans }}
$$

where,

$B_{c h}$ : Bits per one channel.

$N_{c h}$ : Number of channels.

$N_{\text {Orbit }}:$ Number of orbits per day.

$N_{\text {Trans }}$ : Number of data transfers per orbit.

The data packet waveforms, the RS422 driver outputs, are shown in Fig. 5 and the numerical logs forwarded to the $\mathrm{OBC}$ are displayed in Fig. 6 respectively. For converting and displaying the binary data as hexadecimal, the S3Manager

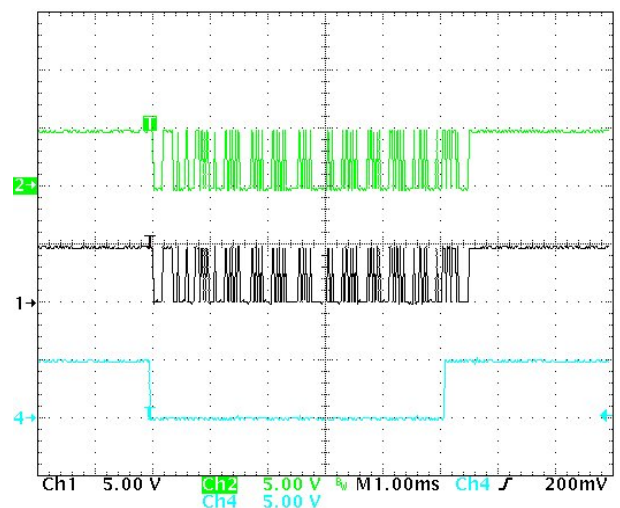

Fig. 5. The data packet waveforms of BCMs. (Ch1) Data packet from primary $\mathrm{BCM}$ to primary $\mathrm{OBC}$ [5V/div.], (Ch2) Data packet from primary BCM to redundant OBC [5V/div.], (Ch4) Flag for transmitting [5V/div.], Time div. : $1 \mathrm{~ms} / \mathrm{div}$

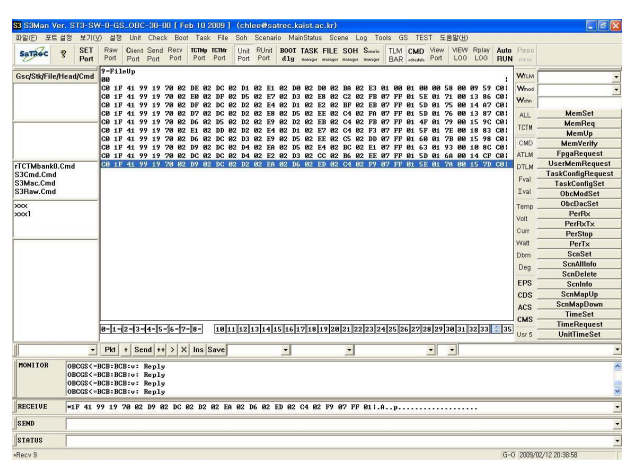

Fig. 6. Numerical logs in S3Manager program.

\begin{tabular}{|c|c|c|c|c|c|c|}
\hline DA & SA & FT & Length & CN & ADC Data & $\begin{array}{c}\text { Checksum } \\
\text { 1Bytes }\end{array}$ \\
\hline
\end{tabular}

\begin{tabular}{|c|c|c|c|c|}
\hline Cell Voltage of $B_{1} \sim B_{8}$ & Battery Charge & Battery Discharge & $\begin{array}{l}\text { Switch Current } \\
\text { of Primary Current of }\end{array}$ \\
\hline
\end{tabular}

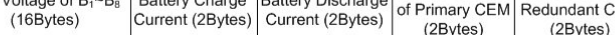

Fig. 7. Packet data format of BCMs.

program (developed on our own) is used.

Fig. 7 shows the packet format created and transmitted by the $\mathrm{BCMs}$

where:

- DA: Destination (OBC or Ground Station) address ID.

- SA: Source (BCM) address ID.

- FT: Frame type.

- Length: Total bytes of the ADC data field.

- CN: Analog telemetry channel number

- ADC data: Digitalized cell voltage, charge/discharge current and current of the CEM switches.

- Checksum: Sum of bytes between the length field and the ADC Data.

The important parameters like the state of charge (SOC) and the state of health ( $\mathrm{SOH})$ [5] depend on the ampere hour (Ah) capability, the battery technology and the type of load connected to the battery pack. They can be estimated using the transmitted data and the parameters provided by the manufacturer at the GS.

2) Cell equalization module (CEM): Fig. 8 shows the modified fly back converter topology for a CEM. Its primary 


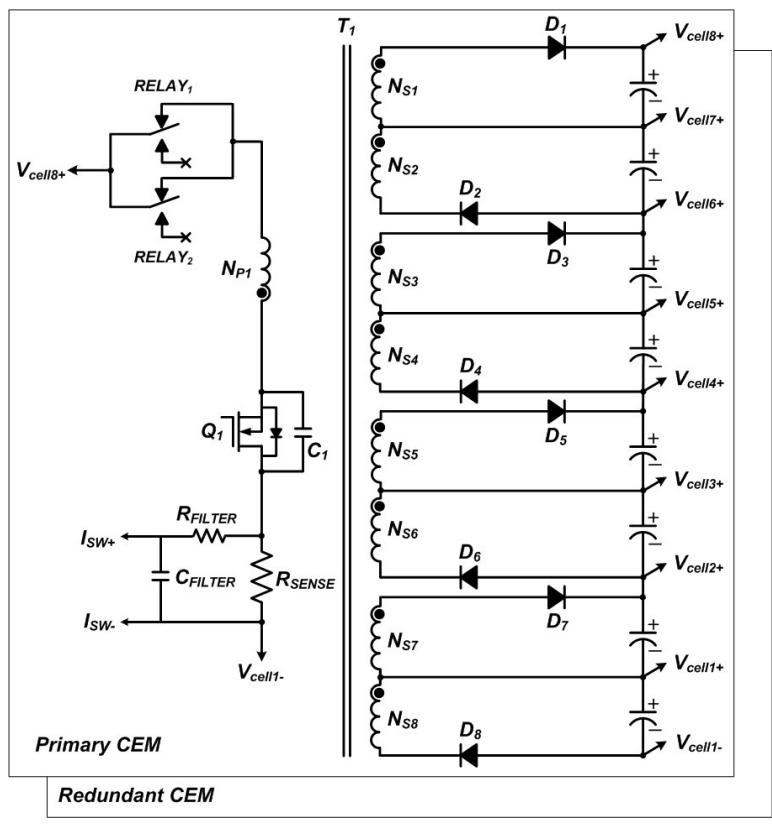

Fig. 8. Circuit diagram of the cell equalization module (CEM).

side is simplified to use only one MOSFET switch and a transformer with multi-winded outputs. The secondary side of converter is improved to reduce the number of connection pins in the secondary bobbin. Similar to the BCMs, an auxiliary circuit is provided in case there is a defect in the primary circuit.

a) Operation of the cell equalization module (CEM):

The operation of each cell balancing circuit is similar to the ones used in the conventional fly-back topology. The energy in the multi-winding flyback converter is stored in the $L_{m}$ when the switch is closed and it is transferred to the load when the switch is open. When the switch is opened, the varying current in the transformer magnetizing inductance, $\left(\Delta I_{L M}\right)_{\text {open }}$ is expressed as:

$$
\left(\Delta I_{L_{m}}\right)_{\text {open }}=\frac{-V_{O}(1-D)}{f_{S} L_{m}} \frac{N_{2}}{N_{1}} .
$$

Since the net change in the inductor current must be zero over one period for steady state operation, in the multiwinding flyback topology, the different output voltage and the cell voltage, cause the different secondary output currents. Therefore, these current differences make the cell voltages equal. The specifications for the CEM design are listed in Table 3.

TABLE III

ELECTRICAL DESIGN SPECIFICATIONS FOR STSAT-3 LIBU

\begin{tabular}{cc}
\hline Item & Value \\
\hline$V_{I N}$ & $28.8 \mathrm{~V}(24 \mathrm{~V} \sim 32.8 \mathrm{~V})$ \\
$V_{\text {out }}$ & $4 \mathrm{~V}$ \\
$I_{\text {out }, R M S}$ & $1 \mathrm{~A}$ \\
$L_{m}$ & $105 \mathrm{uH}$ \\
Turn ratio & $N_{P}: N_{S}=17: 3$ \\
& $N_{P}: 0.4 \varphi / 1$ string \\
Wiring & $N_{S}: 0.4 \varphi / 2$ string \\
\hline
\end{tabular}

b) Power rating design guide: In general, the power rating of a CEM has a very close relation with the equalization time [13], [14]. A higher power rating causes a shorter equalization time. The notations for solving the generalized output power equation are as follows:

$Q_{n}(t) \quad$ Charge quantity of the $n$th cell at time $t$.

$v_{n}(t) \quad$ Voltage of the $n$th cell at time $t$.

$I_{n} \quad$ Constant input current of the $n$th cell.

$I_{\text {in }}, I_{\text {out }} \quad$ Constant input current and output current of the CEM.

$p_{\text {in }}(t), p_{\text {out }}(t) \quad$ Input power and output power of the CEM at time $t$.

$P_{\text {in,avg }}, P_{\text {out }, \text { avg }}$ Average input power and average output power of the CEM.

$\eta \quad$ Overall efficiency of the CEM.

C Capacitance of the Li-ion cell.

To obtain the optimal power rating of the CEM, the following simultaneous equations should be satisfied:

$$
\begin{aligned}
& \frac{1}{7} \sum_{n=2}^{n=8} Q_{n}(t)=Q_{1}(t) \\
& P_{\text {out }, \text { avg }}=\eta P_{\text {in }, \text { avg }} .
\end{aligned}
$$

From (4) and (5), we can see that the charge quantity left in the first cell at the equalization time $t$ is equal to the average charge quantity of the other cells. The overall efficiency of the CEM is $\eta$.

To show one example, we assumed that the $N$ th and $M$ th cells are undercharged within the CEM at the start of the balancing operation. For leading the power equation, three statements are assumed to be true:

1) Transformer leakage inductance can be ignored.

2) Semiconductor devices used on the CEM are ideal.

3) $v_{\text {others }}\left(t_{0}\right)>v_{M}\left(t_{0}\right)>v_{N}\left(t_{0}\right)$.

The output power of the CEM topology flows into the lower cells, which is the balancing current for the equalization. The equalization of the CEM is implemented in two phases. First phase is when the voltage of the $N$ th cell is charged to the $M$ th cell voltage. The second phase is when the $N$ th and $M$ th cell voltages become equal to the other cells' voltages. The varying cell voltages according to each phase are drawn in Fig. 9.

In the first phase, the amount of the average charge $Q_{N}(t)$ and the output power of the CEM, $P_{\text {Nout,avg }}$ to equalize the undercharged $N$ th cell can be expressed as:

$$
\begin{aligned}
Q_{N}\left(t_{1}\right) & =Q_{N}(0)+\left(I_{\text {out }}-I_{\text {in }}\right) \cdot t_{1} \\
P_{\text {Nout }, \text { avg }} & =\frac{1}{t_{1}} \int_{0}^{t_{1}} P_{\text {Nout }}(\tau) d \tau=\frac{1}{t_{1}} \int_{0}^{t_{1}} V_{N}(\tau)\left(I_{\text {out }}-I_{\text {in }}\right) d \tau \\
& =\frac{1}{t_{1}} \int_{0}^{t_{1}}\left(V_{N}(0)+\frac{\left(I_{\text {out }}-I_{\text {in }}\right) \cdot \tau}{C}\right)\left(I_{\text {out }}-I_{\text {in }}\right) d \tau \\
& =\left(V_{N}(0)+\frac{1}{2 C}\left(I_{\text {out }}-I_{\text {in }}\right) \cdot t_{1}\right)\left(I_{\text {out }}-I_{\text {in }}\right) .
\end{aligned}
$$

In second phase, the amount of the average charge $Q_{N}(t), Q_{M}(t)$ and the output power of the CEM, $P_{N o u t, a v g}$ 


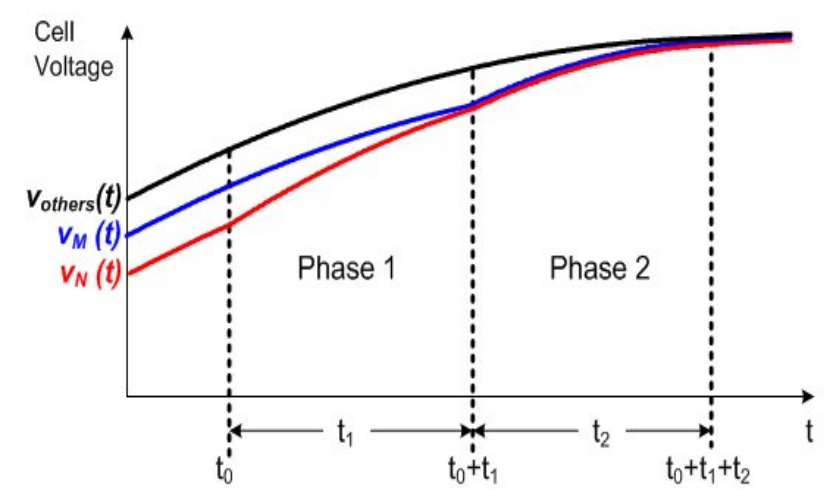

Fig. 9. State diagram of BCMs.

and $P_{\text {Mout,avg }}$ to charge the undercharged $N$ th and $M$ th cell voltages can be solved with the following:

$$
\begin{aligned}
Q_{N}\left(t_{2}\right) & =Q_{N}(0)+\left(I_{\text {out }}-I_{\text {in }}\right) \cdot t_{2} \\
Q_{M}\left(t_{2}\right) & =Q_{M}(0)+\left(I_{\text {out }}-I_{\text {in }}\right) \cdot t_{2} \\
P_{\text {Nout }, \text { avg }} & =\frac{1}{t_{2}} \int_{0}^{t_{2}} P_{\text {Nout }}(\tau) d \tau=\frac{1}{t_{2}} \int_{0}^{t_{2}} V_{N}(\tau)\left(I_{\text {out }}-\frac{I_{\text {in }}}{2}\right) d \tau \\
& =\frac{1}{t_{2}} \int_{0}^{t_{2}}\left(V_{N}(0)+\frac{\left(I_{\text {out }}-\frac{I_{\text {in }}}{2}\right) \cdot \tau}{C}\right)\left(I_{\text {out }}-\frac{I_{\text {in }}}{2}\right) d \tau \\
& =\left(V_{N}(0)+\frac{1}{2 C}\left(I_{\text {out }}-\frac{I_{\text {in }}}{2}\right) \cdot t_{2}\right)\left(I_{\text {out }}-\frac{I_{\text {in }}}{2}\right) \\
P_{\text {Mout }, \text { avg }} & =\frac{1}{t_{2}} \int_{0}^{t_{2}} P_{M o u t}(\tau) d \tau=\frac{1}{t_{2}} \int_{0}^{t_{2}} V_{M}(\tau)\left(I_{\text {out }}-\frac{I_{\text {in }}}{2}\right) d \tau \\
& \left.=\frac{1}{t_{2}} \int_{0}^{t_{2}}\left(I_{\text {out }}-\frac{I_{\text {in }}}{2}\right) \cdot \tau\right)\left(I_{\text {out }}-\frac{I_{\text {in }}}{2}\right) d \tau \\
\left.\left.V_{M}(0)+\frac{I_{\text {in }}}{2}\right) \cdot t_{2}\right)\left(I_{\text {out }}-\frac{I_{\text {in }}}{2}\right) \cdot &
\end{aligned}
$$

The total balancing time necessary for all the cell voltages to become equal is $t_{1}+t_{2}$.

c) Control Algorithm for the CEM: The BCMs monitor the filtered current of the primary switches, disconnect the relays and disable switches when an over current is detected. The BCMs are designed to start driving the PWM IC in the CEM only if the difference between the minimum and maximum cell voltage is greater than the SOC $\pm 5 \%$ (about $0.1 \mathrm{~V})$. The balancing operation is performed when the battery pack is charged in the taper charge mode. A state diagram of the BCMs is presented in Fig. 10.

For controlling a CEM, BCMs have several modes. Each state is explained below:

- IDLE_STATE: This is the unoccupied state. If a difference between the maximum and minimum cell voltage is smaller than SOC $\pm 5 \%$ range, the BCM does not take any action.

- ACTIVE_STATE: When the gap between the cells becomes near the SOC $\pm 5 \%$, the state switches to this mode for CEM selection. At the first operation of the CEM, the primary circuit operates and the flag register

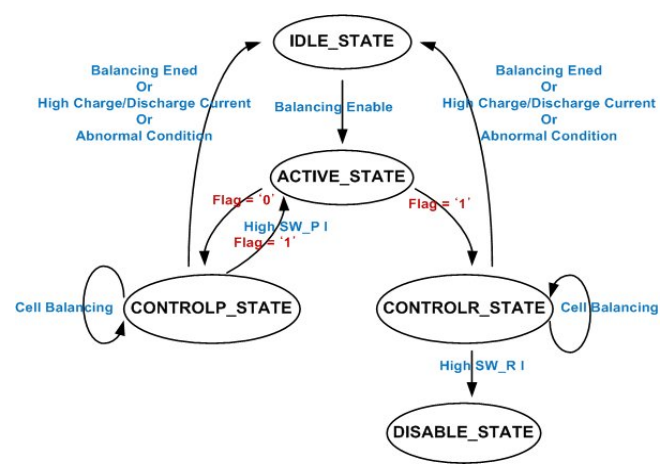

Fig. 10. State diagram of BCMs.

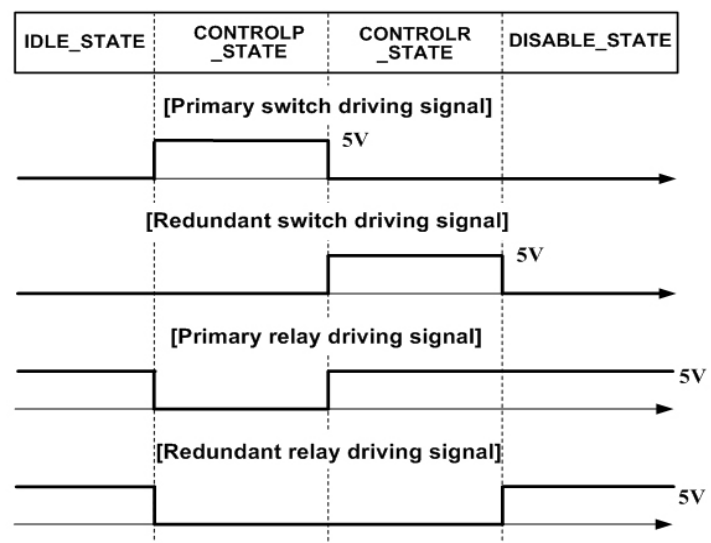

Fig. 11. CEM control signal of BCMs.

is set to ' 0 '. If there are any detected problem like a huge switch current, the auxiliary CEM is turned on and the flag becomes ' 1 '. In the 'ACTIVE_STATE', the BCM decides which CEM should be turned on by referring to the flag of previous balancing operation. The next state is the 'CONTROLP_STATE' when the flag is ' 0 ', and it is the 'CONTROLR_STATE' when the flag is ' 1 '.

- CONTROLP_STATE: Cell voltage equalization is carried out by the primary CEM. In this stage, the state is changed to the 'IDLE_STATE' if the gap is reduced to the SOC $\pm 2.5 \%$, as a result of a battery charge or discharge current. In addition, the state is diverted to the 'IDLE_STATE' if the CEM works abnormally.

- CONTROLR_STATE: Cell voltage equalization is carried out by the auxiliary CEM. The state switchover method to the 'IDLE_STATE' is the same as with the 'CONTROLP_STATE'. In the case of a high switch current being perceived, the state is switched to the 'DISABLE_STATE'.

- DISABLE_STATE: In this stage, the BCM judges the primary and auxiliary CEMs are malfunctioning. All relays are disconnected and the switches are disabled to prevent abnormal operation of the whole system.

The control signals of the BCMs are shown in Fig. 11 according to each state.

d) CEM Test Result: Fig. 12 shows the balancing operation of the CEM. For the test, the $7^{\text {th }}$ cell was arbitrarily discharged so that it would have $120 \mathrm{mV}$ of voltage gap with 


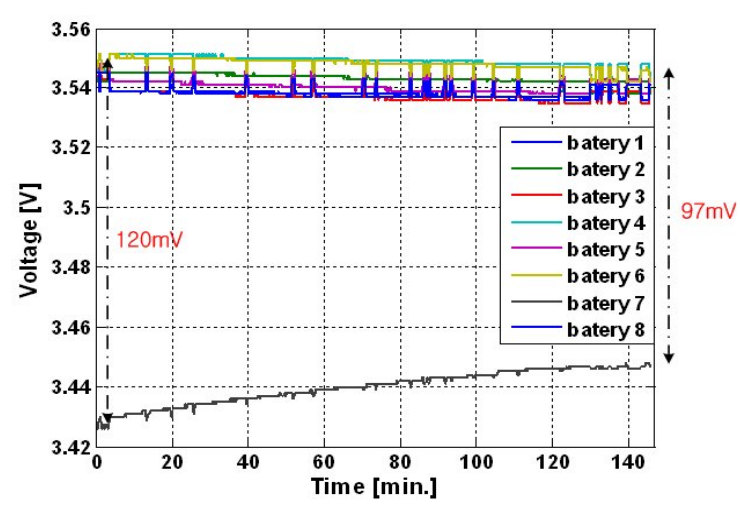

Fig. 12. Balancing operation of CEM.

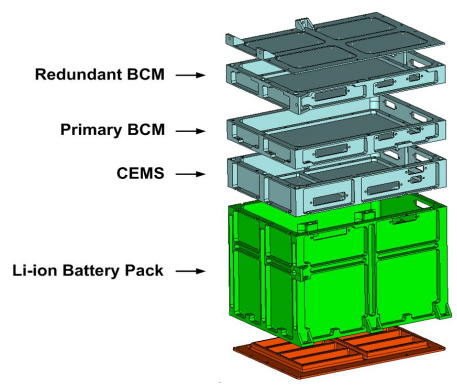

(a) Module stack of LIBU

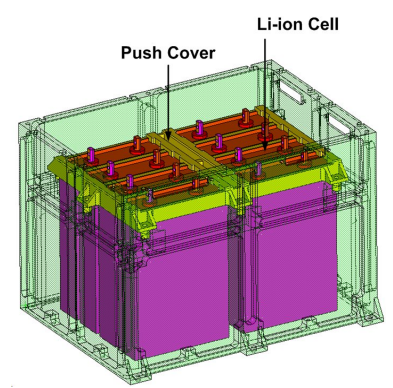

(b) Cell stack of Li-ion battery pack
Fig. 13. Structure of STSAT-3 LIBU.

the highest voltage among the cells. After 2 hours, the gap is reduced to about $100 \mathrm{mV}$.

Fig. 12 shows the balancing operation of the CEM. For the test, the $7^{\text {th }}$ cell was arbitrarily discharged so that it would have $120 \mathrm{mV}$ of voltage gap with the highest voltage among the cells. After 2 hours, the gap is reduced to about $100 \mathrm{mV}$.

The balancing time has some gap with the one obtained from the power rating design guide equation. The gap is caused by different secondary leakage inductances leading different amounts of transferred charge.

\section{Mechanical Design Consideration Of The Bms}

A. The technical specifications of the lithium ion battery unit (LIBU) for the STSAT-3

The battery housing structures for spacecrafts should be able to stand the severe vibration during launching and the extreme temperature variations in orbit. Therefore, the structure design takes into consideration the stresses caused by high pressure, the generation of heat during the charge period and deformation from pressure. Fig. 13 shows the structure of the lithium ion battery unit (LIBU) for the STSAT-3. The LIBU consists of the BMS and the lithium ion battery pack assembled with 8 lithium ion cells.

The LIBU structure consists of a bottom plate, the outer structure surrounding the bottom plate and three more boxes on top of the main body for the PCB as shown in Fig. 13(a). A push cover coated with Teflon is bolted to a rib on the outer structure for observing external impacts. The cells are stacked in slots in the bottom plate and the heat generated by the cells

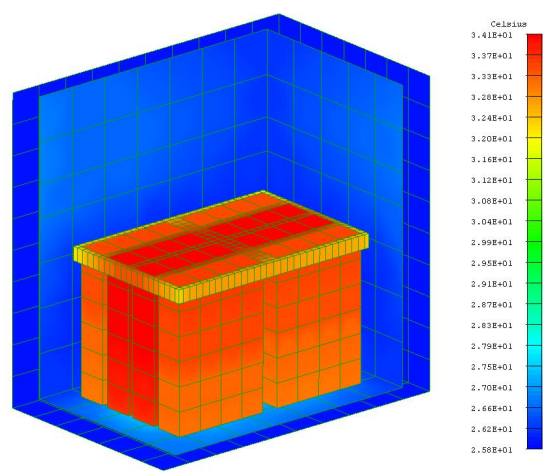

Fig. 14. distribution of temperature in the structure.

is transferred by conduction through the bottom plate and the push cover because there is no convection in space.

The total weight of the LIBU is $12624 \mathrm{~g}$ and the dimensions are $255 \mathrm{~mm}$ [Width] $\times 179.24 \mathrm{~mm}$ [Length] $\times 264 \mathrm{~mm}$ [Height]. The structure is made up of aluminum alloy A17075. The application of the lithium ion battery as an energy storage utensil increased the specific energy of the STSAT-3 LIBU. Its $74 \mathrm{WHr} / \mathrm{kg}$ is about three times as large as the STSAT-2 battery pack.

\section{B. Thermal design}

The performance of the lithium ion battery, like most batteries, is affected by temperature. By considering the thermal characteristics of the lithium ion battery, the battery pack is designed to operate within a safe temperature range.

The lithium ion battery pack generates heat during the charging and discharging periods. When exposed to direct sunlight, the temperature of the battery can be increased to the temperature limit. In order to evaluate the temperature difference between the battery cell and the structure, a thermal analysis of the battery pack was performed.

In the thermal analysis, it was assumed that the internal space is evacuated and that the heat is transferred by conduction only. Fig. 14 shows the temperature distribution of the structure when it is in the thermal steady state condition.

From the analysis, the maximum difference in the temperature between the cell and the structure was expected to be $8.3^{\circ} \mathrm{C}$ and this difference was considered in the system level thermal design.

The qualification model was manufactured and a thermal vacuum test was performed. The thermal vacuum test validates the thermal design of the components and verifies the performance of the vacuum sensitive components. For the STSAT-3 unit level thermal vacuum test, 2.5 thermal vacuum cycles are imposed. The pressure and temperature of the internal chamber and the LIBU are displayed in Fig. 15.

The temperature range is $-7^{\circ} \mathrm{C} \sim 36^{\circ} \mathrm{C}$, which has 10 degrees of margin when compared to the expected range from the on-orbit thermal analysis. The pressure of the internal vacuum chamber is $4.78 \times 10-6$ torr at the end of the thermal vacuum test. During the cycles the electrical functional test was performed at each of the hot and cold soaks and there were no functional defects or errors. 


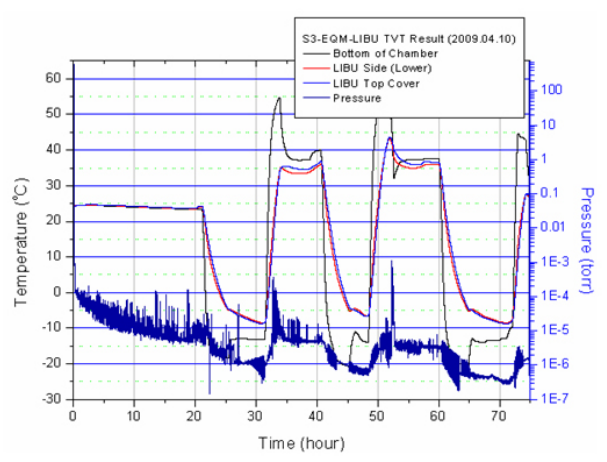

Fig. 15. The pressure and temperature monitoring.

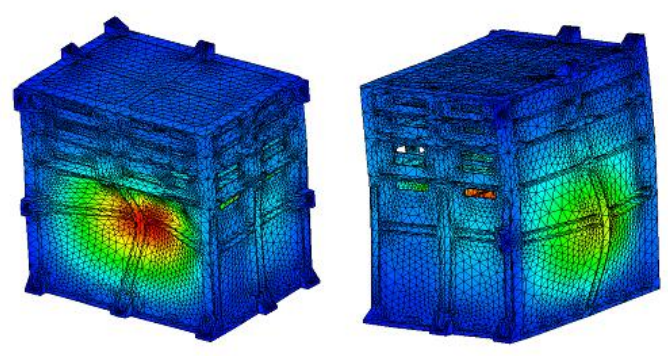

Fig. 16. Simulation result of the first eigenfrequency of LIBU.

\section{Vibration design}

The stresses caused by the launching environment affects the structure. When the external excitation frequencies are coincident with structure's natural frequencies, resonance occurs. The eigenfrequency is an essential characteristic of structure used to define a structure coupling with another structure. Fig.16 shows the structure shape of the STSAT-3 LIBU at the first eigenfrequency.

For the simulation of stress, the finite element analysis model was used. The first eigenfrequency of the LIBU, 1837.9 $\mathrm{Hz}$, is very high when compared to other satellite applications, which are several tens or hundreds of $\mathrm{Hz}$.

In order to verify the robustness of the LIBU structure design, a random vibration test was performed. A mechanical frame was used as a substitute for the satellite frame as shown in Fig. 17 (a). The test was executed by shaking the structure along each axis and then measuring the $1^{\text {st }}$ natural frequency. The vibration is slight for the pre and post vibration tests and sturdy for the main test. The FRF responses from an accelerometer attached at the center of the top cover-edge are displayed in Figs. $17(b) \sim(d)$ and the $1^{\text {st }}$ natural frequency of the LIBU in each phase is displayed in table 4. Before and after the vibration test, the electrical functions were tested for to detect errors caused by the vibration test. However, there were no functional faults.

\section{CONCLUSIONS}

This paper presents the lithium ion battery unit (LIBU) for the STSAT-3 satellite. Using lithium ion cells, the specific energy is enhanced and the number of cells required to generate the same bus voltage is reduced when compared with a $\mathrm{Ni}-\mathrm{Cd}$ battery. The electrical design is introduced to cope with the

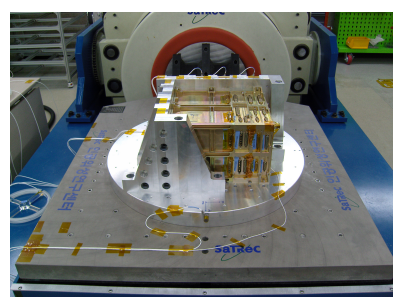

(a) Test facilities set up

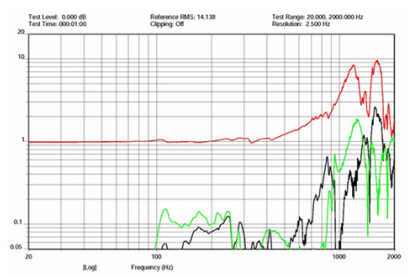

(c) Y-axis vibration data

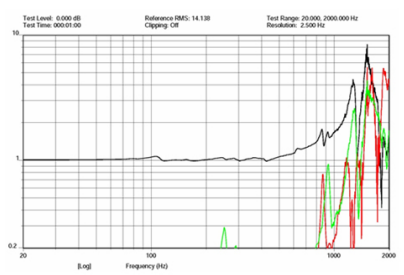

(b) $\mathrm{X}$-axis vibration data

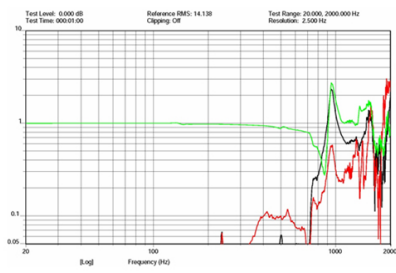

(d) Z-axis vibration data
Fig. 17. The vibration test results.

TABLE IV

THE $1^{\text {st }}$ NATURAL FREQUENCY CHARACTERISTIC IN VIBRATION TEST

\begin{tabular}{cccc}
\hline $1^{\text {st }}$ natural frequency $(\mathrm{Hz})$ & X Axis & Y Axis & Z Axis \\
\hline Low level pre-vibration test & 1672 & 1632 & 965 \\
Main vibration test & 1660 & 1610 & 947 \\
Low level post-vibration test & 1672 & 1632 & 965 \\
\hline
\end{tabular}

sensitivity to over-charge exhibited by lithium ion batteries and to enhance the lifecycles of the batteries. Test results are shown to validate the design. A mechanical structure design to overcome the harsh environment is proposed. We have performed some simulations and safety tests with the qualification model. The behavior of the cells and structure are confirmed from these tests and simulations. Moreover, all these results lend credibility to the use of lithium ion batteries in satellites.

\section{ACKNOWLEDGMENT}

This work was supported by the Science and Technology Satellite 3 (STSAT-3) Development Grant funded by the Satellite Technology Research Center (SaTReC).

\section{REFERENCES}

[1] T. Gonai, T. Kiyokawa, H. Yamazaki, and M. Goto, "Development of the lithium ion battery system for space: report on the result of development of the lithium ion battery system for space," Telecommunications Energy Conference, 2003. INTELEC '03. The 25th International, pp. 234-240, Oct. 2003.

[2] R. Bugga, M. Smart, J. Whitacre, and W. West, "Lithium ion aerospace batteries," Aerospace Conference, 2007 IEEE, pp. 1-7, Mar. 2007.

[3] V. L. Teofilo, M. J. Isaacson, R. L. Higgins, and E. A. Cuellar, "Advanced lithium ion solid polymer electrolyte battery development," IEEE Aerospace and Electronic Magazine, Vol. 14, Issue 11, pp. 43-47, Nov. 1999.

[4] M.J. Isaacson, V.L. Teofilo, "Lithium ion aerospace batteries," Battery Conference on Applications and Advances, 2002. The Seventeenth Annual, pp. 119-122, Jan. 2002.

[5] A. Jossen, V. path, H Doring, and J Garche, "Battery management systems (BMS) for increasing battery life time," 21st 1999 International Telecommunications Energy Conference (INTELEC99), pp. 3-1. Jun. 1999.

[6] J. Chatzakis, K. Kalaitzakis, N. C. Voulgaris, and S. N. Manias, "Designing a new generalized battery management system," IEEE Transactions in Industrial Electronics, Vol. 50, No. 5, pp. 990-999, Oct. 2003. 
[7] K. Shimitzu, N. Shirai, and M. Nihei, "On-board battery management system with SOC indicator," Proc. Int. Electric Vehicle Symp., Vol. 2, pp. 99-104, 1996.

[8] J. M. Andrews and R. H. Johnes, "A VRLA battery management system," Proc. INTELEC, pp. 507-513, 1996.

[9] J. A. Asumadu, and M. Haque, H. Vogel, and C. Willards, "Precision battery management system," Instrumentation and Measurement Technology Conference, Vol. 2, pp. 1317-1320, May 2005.

[10] J. Alzieu, P. Gangol, and H. Smimite, "Development of an on-board charge and discharge management system for electric-vehicle batteries," J. Power Sources, Vol. 53, pp. 327-333, 1995.

[11] W. Retzlaff, "On-board battery diagnostic and charge equalizing system (BADICHEQ)," Proc. 11th Int. Electric Vehicle Symp., Vol. 2, pp. 20.03/1-20.30/12, Sep. 1992.

[12] Z. Noworolski and J.M. Noworolski, "A microcomputer-based UPS battery management system,” Proc. IEEE APEC'91, pp. 475-479, 1991.

[13] Hong-Sun Park, Chong-Eun Kim, Chol-Ho Kim, Gun-Woo Moon, and Joong-Hui Lee, "A modularized charge equalizer for an HEV lithiumion battery string," Industrial Electronics, IEEE Transactions on, Vol. 56, Issue 5, pp. 1464-1476, May 2009.

[14] Chol-Ho Kim, Hong-Sun Park, Chong-Eun Kim, Gun-Woo Moon, and Joong-Hui Lee, "Individual charge equalization converter with parallel primary winding of transformer for series connected lithium-ion battery dtrings in an HEV," Journal of Power Electronics, Vol. 9, Issue 3, pp. 472-480, May 2009.

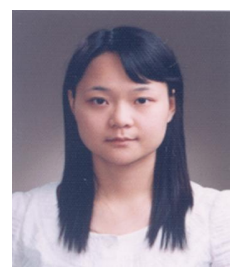

Kyung-Hwa Park was born in Daejeon, Korea, in 1980 She received her B.S. in Electronic Engineering from the Chungnam National University Daejeon, Korea, in 2003 and her M.S. degree in Electrical Engineering from Korean Advanced Institute of Science and Technology (KAIST), Daejeon, Korea, in 2005. Since 2005, she has been working in the Satellite Technology Research Center as a researcher. She is engaged in research on battery management systems and power systems for

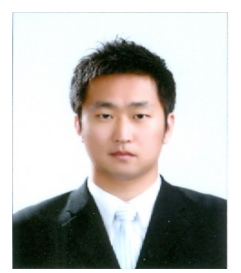

Chol-Ho Kim was born in Iksan, Korea, in 1982. He received his B.S. and M.S. in Electrical Engineering in 2006 and 2008, respectively, from the Korea Advanced Institute of Science and Technology (KAIST), Deajeon, Korea, where he is currently working toward his Ph.D. His research interests include the design and control of HEV BMS, charge equalization converters, and low voltage high current DC/DC converters. Mr. Kim is a student member of IEEE and a member of the Korea Institute of Power Electronics (KIPE).

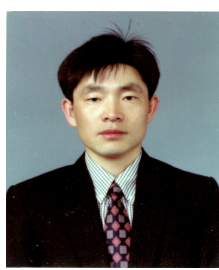

Hee-Keun Cho received his $\mathrm{Ph}$. D. in Mechanical Engineering from the University of Wisconsin-Madison, WI, USA, in 2006. He has over 40 publications in theoretical and numerical analysis; composite, finite element analysis, optimization, and functionally graded materials. Dr. Cho is currently working at the Satellite Technology Research Center of KAIST, Deajeon, Korea, and is involved in the Satellite Development Project (STSAT-3) administered by the Korean government.

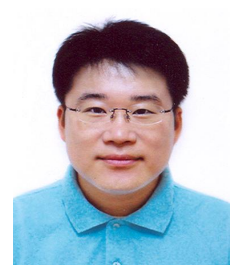

Joung-Ki Seo was born in Seoul, Korea, in 1975. He received his B.S. and M.S. in Mechanical Engineering from KAIST, Deajeon, Korea, in 1998 and 2005, respectively. Since 2005, he has been with the faculty of the SaTReC at KAIST as a Research Professor. He is engaged in a mechanical design group for satellite systems, especially thermal design. He is participating in several satellite programs as a thermal designer in Korea. 